Document downloaded from:

http://hdl.handle.net/10251/120924

This paper must be cited as:

Xu, S.; Benítez López, J. (2018). Existence Criteria and Expressions of the (b, c)-Inverse in Rings and Their Applications. Mediterranean Journal of Mathematics. 15(1). https://doi.org/10.1007/s00009-017-1056-x

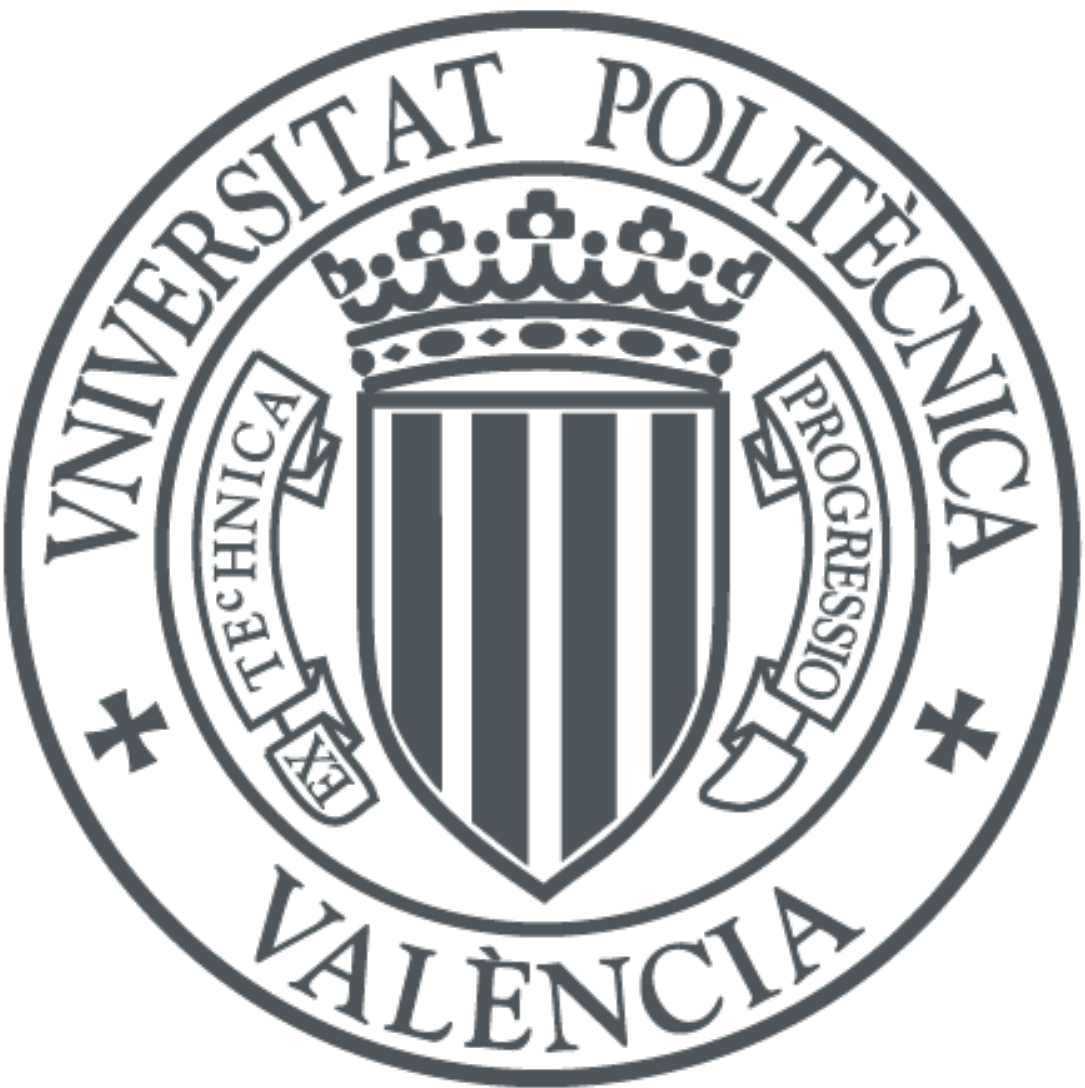

The final publication is available at

https://doi.org/10.1007/s00009-017-1056-x

Copyright Springer-Verlag

Additional Information 


\title{
Existence criteria and expressions of the $(b, c)$-inverse in rings and their applications
}

\author{
Sanzhang $\mathrm{Xu}{ }^{*}$ and Julio Benítez ${ }^{\dagger}$
}

\begin{abstract}
Let $R$ be a ring. Existence criteria for the $(b, c)$-inverse are given.We present explicit expressions for the $(b, c)$-inverse by using inner inverses. We answer the question when the $(b, c)$-inverse of $a \in R$ is an inner inverse of $a$. As applications, we give a unified theory of some well-known results of the $\{1,3\}$-inverse, the $\{1,4\}$-inverse, the Moore-Penrose inverse, the group inverse and the core inverse.
\end{abstract}

Key words: $(b, c)$-inverse, inner inverse, the inverse along an element, annihilator.

AMS subject classifications: 16W10, 15A09.

\section{Introduction}

Throughout this paper, $R$ denotes a unital ring. In [7, Definition 1.3], Drazin introduced a new class of outer inverses in the setting of semigroups, namely, the $(b, c)$-inverse. Let $a, b, c \in R$. We say that $y \in R$ is the $(b, c)$-inverse of $a$ if we have

$$
y \in(b R y) \cap(y R c), y a b=b \text { and } c a y=c .
$$

If such $y \in R$ exists, then it is unique and denoted by $a^{(b, c)}$. The $(b, c)$-inverse is a generalization of the Moore-Penrose inverse, the Drazin inverse, the group inverse and the core inverse. Many existence criteria and properties of the $(b, c)$-inverse can be found in $[3,4,7,8,12,13,17,19,20,21]$ etc. In [7, Definition 6.2 and 6.3], Drazin introduced the hybrid $(b, c)$-inverse and the annihilator $(b, c)$-inverse of $a$. We call that $y \in R$ is the hybrid $(b, c)$-inverse of $a$ if we have yay $=y, y R=b R$ and $y^{\circ}=c^{\circ}$. We call that $y \in R$ is the annihilator $(b, c)$-inverse of $a$ if we have yay $=y,{ }^{\circ} y={ }^{\circ} b$ and $y^{\circ}=c^{\circ}$. By [7, Theorem $6.4]$, if the the hybrid $(b, c)$-inverse (resp. the annihilator $(b, c)$-inverse) of $a$ exists, then it is unique.

In [14], Mary introduced a new type of generalized inverse, namely, the inverse along an element. This inverse depends on Green's relations [9]. Let $a, d \in R$. We say that $a$ is invertible along $d$ if there exists $y \in R$ such that

$$
y a d=d=d a y, y R \subseteq d R \text { and } R y \subseteq R d .
$$

The inverse along an element extends some known generalized inverses, for example, the group inverse, the Drazin inverse and the Moore-Penrose inverse. Many existence criteria of the inverse along an element can be found in $[14,15]$ etc. By the definition of the inverse along $d$, we have that $a$ is invertible along $d$ if and only if $a$ is $(d, d)$-invertible. The unique element $y$ (if exists) satisfying (1.2) is denoted $a^{\sim d}$.

The following notations $a R=\{a x \mid x \in R\}, R a=\{x a \mid x \in R\},{ }^{\circ} a=\{x \in R \mid x a=0\}$ and $a^{\circ}=\{x \in R \mid a x=0\}$ will be used in the sequel. An involutory ring $R$ means that

\footnotetext{
${ }^{*}$ Sanzhang Xu (Corresponding author E-mail: xusanzhang5222@126.com): School of Mathematics, Southeast University, Nanjing 210096, China.

${ }^{\dagger}$ Julio Benítez (E-mail: jbenitez@mat.upv.es): Universitat Politècnica de València, Instituto de Matemática Multidisciplinar, Valencia, 46022, Spain.
} 
$R$ is a unital ring with involution, i.e., a ring with unity 1 , and a mapping $a \mapsto a^{*}$ in $R$ that satisfies $\left(a^{*}\right)^{*}=a,(a b)^{*}=b^{*} a^{*}$ and $(a+b)^{*}=a^{*}+b^{*}$, for all $a, b \in R$. While the basic definitions have no need of it, to discuss the Moore-Penrose inverse we need to also assume that $R$ has an involution. The notations of the $\{1,3\}$-inverse, the $\{1,4\}$ inverse, the Moore-Penrose inverse, and the group inverse can be found in $[2,5,6,20]$. The notation of the core inverse can be found in [1].

\section{$2 \quad$ Preliminaries}

In this section, we will collect and present some useful preliminaries, which will be used in the sequel.

Lemma 2.1. Let $a \in R$. Then

(1) $\left[10\right.$, p.201] $a \in R^{\{1,3\}}$ with $x \in a\{1,3\}$ if and only if $x^{*} a^{*} a=a$;

(2) $\left[10\right.$, p.201] $a \in R^{\{1,4\}}$ with $y \in a\{1,4\}$ if and only if $a a^{*} y^{*}=a$;

(3) [11, Theorem 2] $a \in R^{\{1,3\}}$ if and only if $R=R a^{*} \oplus^{\circ} a$;

(4) [11, Theorem 3] $a \in R^{\{1,4\}}$ if and only if $R=a^{*} R \oplus a^{\circ}$.

The subset of $R$ of group invertible elements will be denoted by $R^{\#}$, and if $a \in R^{\#}$, then $a^{\#}$ denotes the group inverse of $a$.

Lemma 2.2. Let $a \in R$. Then

(1) $\left[10\right.$, Proposition 7] $a \in R^{\#}$ if and only if $R=a R \oplus a^{\circ}$.

(2) [10, Proposition 7] $a \in R^{\#}$ if and only if $R=R a \oplus^{\circ} a$.

(3) [5, Proposition 8.22] $a \in R^{\#}$ if and only if $a^{2} x=a$ and $y a^{2}=a$ both have solutions.

An element $a \in R$ is regular if and only if $a \in a R a$. The subset of $R$ composed of regular elements will be denoted by $R^{\cap}$.

Lemma 2.3. [16, Lemma 8] Let $a, b \in R$. Then:

(1) $a R \subseteq b R$ implies ${ }^{\circ} b \subseteq{ }^{\circ} a$ and the converse is valid whenever $b$ is regular;

(2) $R a \subseteq R b$ implies $b^{\circ} \subseteq a^{\circ}$ and the converse is valid whenever $b$ is regular.

Lemma 2.4. [21, Lemma 3.2] Let $a, b \in R$. Then:

(1) Let $a R=b R$. If a is regular, then $b$ is regular;

(2) Let $R a=R b$. If $a$ is regular, then $b$ is regular.

Lemma 2.5. [21, lemma 3.1] Let $a, y \in R$ with yay $=y$. Then:

(1) $y a R=y R$;

(2) $R a y=R y$;

(3) $a^{\circ} \cap y R=a^{\circ} \cap y a R=\{0\} ;$ 
(4) ${ }^{\circ} a \cap R y={ }^{\circ} a \cap R a y=\{0\}$.

Lemma 2.6. [14, Theorem 7] Let $a, d \in S$. Then the following statements are equivalent:

(1) $a^{(d, d)}$ exists

(2) $d R \subseteq d a R$ and $(d a)^{\#}$ exists;

(3) $R d \subseteq R a d$ and $(a d)^{\#}$ exists.

In this case,

$$
a^{(d, d)}=d(a d)^{\#}=(d a)^{\#} d .
$$

Lemma 2.7. [15, Theorem 2.2] Let $a, d \in S$. Then a is invertible along $d$ if and only if $d R=d a d R$ and $R d=R d a d$.

Lemma 2.8. [7, Theorem 2.1 (ii) and Proposition 6.1] Let $a, b, c \in R$. Then $y \in R$ is the $(b, c)$-inverse of $a$ if and only if $y a y=y, y R=b R$ and $R y=R c$.

Lemma 2.9. [7, Theorem 2.2] Let $a, b, c \in R$. Then there exists at least one $(b, c)$-inverse of $a$ if and only if $b \in R c a b$ and $c \in c a b R$.

Lemma 2.10. [21, Proposition 3.3] Let $a, b, c \in R$. If there exists a $(b, c)$-inverse of $a$, then $c a b, b$ and $c$ are regular.

It is easy to prove that $a \in R$ has a $(b, c)$-inverse then necessarily $\{b, c\} \subseteq R^{\cap}$. In fact, let $y=a^{(b, c)}$. Since $y \in(b R y) \cap(y R c)$, exists $z \in R$ such that $y=b z y$, and now $b=y a b=b z y a b \in b R b$. The proof of $c \in c R c$ is similar.

Lemma 2.11. [12, Proposition 2.7] Let $a, b, c \in R$. Then the following are equivalent:

(1) a is $(b, c)$-invertible;

(2) $c \in R^{\cap}, a^{\circ} \cap b R=\{0\}$ and $R=a b R \oplus c^{\circ}$;

(3) $b \in R^{\cap},{ }^{\circ} a \cap R c=\{0\}$ and $R=R c a \oplus^{\circ} b$.

By [12, Theorem 2.9] and the definitions of hybrid $(b, c)$-inverse and annihilator $(b, c)$ inverse, we have the following lemma.

Lemma 2.12. Let $a, b, c, y \in R$. Then the following are equivalent:

(1) $y$ is the $(b, c)$-inverse of $a$;

(2) $c \in R^{\cap}, y$ is the hybrid $(b, c)$-inverse of $a$;

(3) $b, c \in R^{\cap}, y$ is the annihilator $(b, c)$-inverse of $a$.

In [12, Theorem 2.11], the authors gave a generalization of [20, Theorem 2.1]. By Lemma 2.2 and [12, Theorem 2.11], we have the following lemma.

Lemma 2.13. Let $a, b, c, d \in R, d R=b R$ and $d^{\circ}=c^{\circ}$. If $a$ is $(b, c)$-invertible, then $a d$ and da are group invertible. Furthermore, we have

$$
a^{(b, c)}=a^{\sim d}=d(a d)^{\#}=(d a)^{\#} d .
$$

Proof. Since $a$ is $(b, c)$-invertible, then $b$ and $c$ are regular by Lemma 2.10. By $d R=b R$ and Lemma 2.4, we have $d$ is regular, thus $d^{\circ}=c^{\circ}$ if and only if $R d=R c$. Let $x$ be the $(b, c)$-inverse of $a$. Then $x a x=x, x R=b R$ and $R x=R c$. Thus $x a x=x, x R=d R$ and $R x=R d$, which implies that $a$ is invertible along $d$ by [14, Lemma 3]. Therefore, the proof is finished by Lemma 2.6. 


\section{Existence criteria of the $(b, c)$-inverses and its applications}

In this section, necessary and sufficient conditions of the $(b, c)$-invertibility are given and we present explicit expressions for the $(b, c)$-inverse by using inner inverses. In Theorem 3.11, we will give a generalization of the well-known results in [20, Theorem 2.1]. We answer the question when the $(b, c)$-inverse of $a$ is an inner inverse of $a$. In Theorem 3.14 and Theorem 3.15, we will give a unified theory of some well-known results of the $\{1,3\}$-inverse, the $\{1,4\}$-inverse, the Moore-Penrose inverse, the group inverse and the core inverse.

Theorem 3.1. Let $a, b, c \in R$. Then the following are equivalent:

(1) $a$ is $(b, c)$-invertible;

(2) $c \in R^{\cap},(a b)^{\circ}=b^{\circ}$ and $R=a b R \oplus c^{\circ}$;

(3) $b \in R^{\cap},{ }^{\circ}(c a)={ }^{\circ} c$ and $R=R c a \oplus^{\circ} b$.

Proof. (1) $\Rightarrow(2)$. By Lemma 2.11, we have $c \in R^{\cap}$ and $R=a b R \oplus c^{\circ}$. Let $y$ be the $(b, c)$-inverse of $a$, then $b=y a b$. For arbitrary $u \in(a b)^{\circ}$, we have $b u=y a b u=0$, which implies that $(a b)^{\circ} \subseteq b^{\circ}$. Thus $(a b)^{\circ}=b^{\circ}$ because $b^{\circ} \subseteq(a b)^{\circ}$ is trivial.

$(2) \Rightarrow(1)$. Let $v \in a^{\circ} \cap b R$. Then $a v=0$ and $v=b r$ for some $r \in R$. Thus $a b r=a v=0$, that is $r \in(a b)^{\circ}$. The condition $(a b)^{\circ}=b^{\circ}$ gives that $r \in b^{\circ}$, then $v=b r=0$. Therefore, $a$ is $(b, c)$-invertible by Lemma 2.11 .

The proof of $(1) \Leftrightarrow(3)$ is similar to the proof of $(1) \Leftrightarrow(2)$.

The notion of core inverse for a complex matrix was introduced in [1]. In [18], the core inverse of a complex matrix was generalized to rings with an involution. More precisely, let $a, x \in R$, if

$$
a x a=a, x R=a R \text { and } R x=R a^{*},
$$

then $x$ is called a core inverse of $a$. If such an element $x$ exists, then it is unique and denoted $a^{\boxplus}$. The subset of $R$ composed of core invertible elements is denoted by $R^{\boxplus}$. Also, in [18] the authors defined a related inner inverse in a ring with an involution. If $a \in R$, then $x \in R$ is called a dual core inverse of $a$ if

$$
a x a=a, x R=a^{*} R \text { and } R x=R a .
$$

If such an element $x$ exists, then it is unique.

Let $a \in R$. By [7, p.1910], we have that $a$ is Moore-Penrose invertible if and only if $a$ is $\left(a^{*}, a^{*}\right)$-invertible, $a$ is Drazin invertible if and only if exists $k \in \mathbb{N}$ such that $a$ is $\left(a^{k}, a^{k}\right)$ invertible and $a$ is group invertible if and only if $a$ is $(a, a)$-invertible, By [18, Theorem 4.4], we have the $\left(a, a^{*}\right)$-inverse coincides with the core inverse of $a$ and the $\left(a^{*}, a\right)$-inverse coincides with the dual core inverse of $a$. Thus, by Theorem 3.1, we can get corresponding results of the Moore-Penrose inverse, Drazin inverse, core inverse and dual core inverse. Leaving the deeper details to the reader to research.

The following three lemmas will be useful in the sequel.

Lemma 3.2. Let $a, b, c \in R$ such that $c a b$ is regular. Let $(c a b)^{-}$be an arbitrary element of $(c a b)\{1\}$ and $x=b(c a b)^{-}$c. Then the following are equivalent:

(1) $x a x=x$ and $b R=x R$;

(2) $x a x=x$ and $b R \subseteq x R$; 
(3) $R b=R c a b$;

(4) $b \in R^{\cap}$ and $b^{\circ}=(c a b)^{\circ}$.

Proof. (1) $\Rightarrow(2)$ is trivial.

$(2) \Rightarrow(3)$. Suppose that $x a x=x$ and $b R \subseteq x R$. Then $b=x a b=b(c a b)^{-} c a b \in R c a b$, thus $R b=R c a b$.

$(3) \Rightarrow(1)$. Since $R b=R c a b$ and $c a b$ is regular, then

$$
b=b(c a b)^{-} c a b=\left[b(c a b)^{-} c\right] a b=x a b .
$$

By (3.1) and $x=b(c a b)^{-} c$, we have

$$
x a x=x a b(c a b)^{-} c=b(c a b)^{-} c=x, x R \subseteq b R \text { and } b R \subseteq x R .
$$

Thus, $x a x=x$ and $b R=x R$.

$(3) \Leftrightarrow(4)$. Since $(1) \Leftrightarrow(3)$ and $x$ is regular, we have that $b$ is regular by Lemma 2.4. Thus $(3) \Leftrightarrow(4)$ by Lemma 2.3 and the regularity of $c a b$.

The following lemma is the corresponding result of Lemma 3.2.

Lemma 3.3. Let $a, b, c \in R$ such that $c a b$ is regular. Let $(c a b)^{-}$be an arbitrary element of $(c a b)\{1\}$ and $x=b(c a b)^{-} c$. Then the following are equivalent:

(1) $x a x=x$ and $R x=R c$;

(2) $x a x=x$ and $R c \subseteq R x$;

(3) $c R=c a b R$;

(4) $c \in R^{\cap}$ and ${ }^{\circ} c={ }^{\circ}(c a b)$.

Lemma 3.4. Let $a, b, c \in R$ such that $c a b$ is regular. Let $(c a b)^{-}$be an arbitrary element of $(c a b)\{1\}$ and $x=b(c a b)^{-} c$. Then the following are equivalent:

(1) $x a x=x$ and $x^{\circ}=c^{\circ}$;

(2) $x a x=x$ and $x^{\circ} \subseteq c^{\circ}$;

(3) $c R=c a b R$.

Proof. (1) $\Rightarrow(2)$ is trivial.

$(2) \Rightarrow(3)$. Suppose that $x a x=x$ and $x^{\circ} \subseteq c^{\circ}$. Then $c=c a x=c a b(c a b)^{-} c \in c a b R$, thus $c R=c a b R$.

$(3) \Rightarrow(1)$. Since $c R=c a b R$ and $c a b$ is regular, then

$$
c=c a b(c a b)^{-} c=c a\left[b(c a b)^{-} c\right]=c a x .
$$

By (3.2) and $x=b(c a b)^{-} c$, we have

$$
x a x=b(c a b)^{-} c a x=b(c a b)^{-} c=x .
$$

Let $u \in c^{\circ}$. Then $x u=b(c a b)^{-} c u=0$, that is $c^{\circ} \subseteq x^{\circ}$. Let $v \in x^{\circ}$. Then $c v=c a x v=0$ by (3.2), that is $x^{\circ} \subseteq c^{\circ}$. Thus, $x a x=x$ and $x^{\circ}=c^{\circ}$. 
Thus, by Lemma 2.12, Lemma 3.2 and Lemma 3.4, we have the following theorem, in which we give an explicit expression for the $(b, c)$-inverse, which reduces to the inner inverse.

Theorem 3.5. Let $a, b, c \in R$ such that $c a b$ is regular. Let $(c a b)^{-}$be an arbitrary element of $(c a b)\{1\}$ and $x=b(c a b)^{-} c$. Then the following are equivalent:

(1) $x$ is the $(b, c)$-inverse of $a$;

(2) $x a x=x, b R \subseteq x R$ and $x^{\circ} \subseteq c^{\circ}$;

(3) $b^{\circ}=(c a b)^{\circ}$ and $c R=c a b R$.

By the fact that if $a \in R$ is invertible along $d$ if and only if $a$ is $(d, d)$-invertible, we have the following corollary.

Corollary 3.6. Let $a, d \in R$ such that dad is regular. Let $(d a d)^{-}$be an arbitrary element of $(d a d)\{1\}$ and $x=d(d a d)^{-} d$. Then the following are equivalent:

(1) $x$ is the inverse along $d$ of $a$;

(2) $x a x=x, d R \subseteq x R$ and $x^{\circ} \subseteq d^{\circ}$;

(3) $d^{\circ}=(d a d)^{\circ}$ and $d R=d a d R$.

In [8, Definition 1.2] and [13, Definition 2.1], the authors introduced the one-sided $(b, c)$-inverse in rings. Let $a, b, c \in R$. We call that $x \in R$ is a left $(b, c)$-inverse of $a$ if we have

$$
R x \subseteq R c \text { and } x a b=b .
$$

We call that $y \in R$ is a right $(b, c)$-inverse of $a$ if we have

$$
y R \subseteq b R \text { and } c a y=c .
$$

Lemma 3.7. [13, Proposition 2.8] Let $a, b, c \in R$. Then $y$ is a left $(b, c)$-inverse of $a$ if and only if $y^{*}$ is a right $\left(c^{*}, b^{*}\right)$-inverse of $a^{*}$.

Theorem 3.8. Let $a, b, c \in R$ such that $c a b$ is regular. Let $(c a b)^{-}$be an arbitrary element of $(c a b)\{1\}$. Then

(1) if a is left $(b, c)$-invertible, then a general solution of the left $(b, c)$-inverse of a is

$$
b(c a b)^{-} c+v\left[1-c a b(c a b)^{-}\right] c,
$$

where $v \in R$ is arbitrary;

(2) if a is right $(b, c)$-invertible, then a general solution of the right $(b, c)$-inverse of a is

$$
b(c a b)^{-} c+b\left[1-(c a b)^{-} c a b\right] u,
$$

where $u \in R$ is arbitrary. 
Proof. (1). Let $x$ be a left $(b, c)$-inverse of $a$. Then we have $x a b=b$ and $R x \subseteq R c$. Thus $x=s c$ for some $s \in R$ and $b=x a b=s c a b$. A general solution of $b=s c a b$ is

$$
b(c a b)^{-}+v\left[1-c a b(c a b)^{-}\right],
$$

where $v \in R$ is arbitrary. Let $y=b(c a b)^{-} c+v\left[1-c a b(c a b)^{-}\right] c$. Next we will check $y$ is a left $(b, c)$-inverse of $a$.

$$
y a b=b(c a b)^{-} c a b+v\left[1-c a b(c a b)^{-}\right] c a b=b(c a b)^{-} c a b .
$$

By Lemma 3.2 and [13, Theorem 2.6], we have $R b=R c a b$. Since $c a b$ is regular, then the condition $R b=R c a b$ implies $b=r c a b=r c a b(c a b)^{-} c a b=b(c a b)^{-} c a b$, thus $b=y a b$ by (3.5). Thus $y$ is a left $(b, c)$-inverse of $a$ by $R y \subseteq R c$ is trivial.

(2) follows from (1) and Lemma 3.7.

Theorem 3.9. Let $a, b, c \in R$. If $a$ is both left and right $(b, c)$-invertible, then the left inverse of $a$ and the right inverse of a are unique. Moreover, the left $(b, c)$-inverse of a coincides with the right $(b, c)$-inverse.

Proof. Let $x$ be a left $(b, c)$-inverse of $a$ and $y_{1}$ be a right $(b, c)$-inverse of $a$. Then we have $R x \subseteq R c, x a b=b, y_{1} R \subseteq b R$ and $c a y_{1}=c$. Thus $x=r c$ and $y_{1}=b s$ for some $r, s \in R$. Therefore,

$$
\begin{aligned}
x & =r c=r c a y_{1}=x a y_{1} \\
y_{1} & =b s=x a b s=x a y_{1} .
\end{aligned}
$$

That is $x=y_{1}$. If $y_{2}$ is a another right $(b, c)$-inverse of $a$, in a similar manner, we have $x=y_{2}$. Then $y_{1}=y_{2}$ by $x=y_{1}$ and $x=y_{2}$, that is the right $(b, c)$-inverse of $a$ is unique. In a similar way, the left $(b, c)$-inverse is unique, and by the previous reasoning, these two inverses are equal.

Theorem 3.10. Let $a, b, c \in R$. Then the following are equivalent:

(1) a is $(b, c)$-invertible;

(2) $b, c \in R \cap,{ }^{\circ} c={ }^{\circ}(c a b), R=R c \oplus^{\circ}(a b)$, and $R b=R a b$;

(3) $b, c \in R^{\cap}, b^{\circ}=(c a b)^{\circ}, R=b R \oplus(c a)^{\circ}$, and $c R=c a R$;

(4) $c a b \in R^{\cap}, R c \subseteq R b(c a b)^{-}$c for all $(c a b)^{-} \in(c a b)\{1\}, R=R c \oplus^{\circ}(a b)$, and $R b=R a b$;

(5) $c a b \in R^{\cap}, b R \subseteq b(c a b)^{-} c R$ for all $(c a b)^{-} \in(c a b)\{1\}, R=b R \oplus(c a)^{\circ}$, and $c R=c a R$.

Proof. $(1) \Rightarrow(2)$. Suppose that $y$ is the $(b, c)$-inverse of $a$. Then the condition $y a b=b$ implies that that $R b=R a b$. By Lemma 2.3, Lemma 2.9 and Lemma 2.10, we have $b, c, c a b \in R^{\cap}$ and ${ }^{\circ} c={ }^{\circ}(c a b)$. Since $1=a y+(1-a y), a y \in R y=R c$ by Lemma 2.8 and $(1-a y) a b=a b-a y a b=a b-a b=0$, thus $R=R c+{ }^{\circ}(a b)$. Let $w \in R c \cap{ }^{\circ}(a b)$. Then $w=t c$ and $w a b=0$ for some $t \in R$. Thus $t c a b=w a b=0$, that is $t \in{ }^{\circ}(c a b)$. By ${ }^{\circ} c={ }^{\circ}(c a b)$, we have $t c=0$, i.e. $w=0$. Therefore, $R=R c \oplus^{\circ}(a b)$.

$(2) \Rightarrow(1)$. The condition $R=R c \oplus^{\circ}(a b)$ implies that $1=u c+v$, where $u \in R$ and $v \in{ }^{\circ}(a b)$. Then $a b=u c a b+v a b=u c a b \in R c a b$, then $R b=R c a b$ by $R b=R a b$. Since $b \in R^{\cap}$ and $R b=R c a b$, then $c a b$ is regular by Lemma 2.4. Let $(c a b)^{-} \in(c a b)\{1\}$ and $x=b(c a b)^{-} c$. By Theorem 3.5, we have that $x$ is the $(b, c)$-inverse of $a$. 
$(2) \Rightarrow(4)$. Since $(1) \Leftrightarrow(2)$, it is easy to check (4) by the proof of $(2) \Rightarrow(1)$.

$(4) \Rightarrow(2)$. By the proof of $(2) \Rightarrow(1)$, we have $R b=R c a b$, then $b$ is regular by $c a b \in R^{\cap}$ and Lemma 2.4. Let $(c a b)^{-} \in(c a b)\{1\}$ and $x=b(c a b)^{-} c$. Since $R b=R c a b$, we have $x a x=x$ by Lemma 3.2. The condition $R c \subseteq R b(c a b)^{-} c$ implies that $R c=R x$, thus $c$ is regular by $x a x=x$. The proof is finished by Theorem 3.5.

The proofs of $(1) \Leftrightarrow(3)$ and $(3) \Leftrightarrow(5)$ are similar to the proofs of $(1) \Leftrightarrow(2)$ and $(2) \Leftrightarrow(4)$, respectively.

In [12, Theorem 2.11], the authors gave a generalization of [20, Theorem 2.1]. In the following theorem, we will present a generalization of [12, Theorem 2.11], which reduces to the $(b, c)$-inverse of $a$. As applications of the Lemma 3.11, we have that [12, Theorem 2.14] and $[12$, Theorem 2.13] can be generalized.

Lemma 3.11. [4, Remark 2.2 (i)] Let $a, d, u, v \in R$. If $b R=u R$ and $R c=R v$, then $a$ is $(b, c)$-invertible if and only if $a$ is $(u, v)$-invertible. In this case, we have $a^{(b, c)}=a^{(u, v)}$.

Proof. Let $b R=u R$ and $R c=R v$. Suppose that $y$ is $(b, c)$-inverse of $a$, then yay $=y$, $y R=b R$ and $R y=R c$ by Lemma 2.8. By $b R=u R$ and $R c=R v$, we have $y a y=y$, $y R=u R$ and $R y=R v$, that is $y$ is $(u, v)$-inverse of $a$. The opposite implication can be proved in a similar manner.

Corollary 3.12. Let $a, d, c \in R$. If $a$ is $(b, c)$-invertible, $b R=e b R$ and $R c=R c f$, then $a$ is $(e b, c f)$-invertible. In this case, we have $a^{(b, c)}=a^{(e b, c f)}$.

If we let $u=v=d$ in Lemma 3.11, then by Lemma 2.6 and Lemma 2.7 and Lemma 2.11, we have that [12, Theorem 2.14] is a corollary of Lemma 3.11. It is wellknown that if $a \in R$ is invertible along $d$ if and only if $a$ is $(d, d)$-invertible. Thus we have that [12, Theorem 2.13] is a corollary of Lemma 3.11.

By Lemma 2.6 and [12, Theorem 2.13], we have the following remark. Since $a \in R$ is invertible along $d$ if and only if $a$ is $(d, d)$-invertible, a natural question is when an element invertible along $d$ is $(b, c)$-invertible, the following remark answers this question.

Remark 3.13. Let $a, b, c, d \in R$ with $b, c$ are regular, $d R=b R$ and $d^{\circ}=c^{\circ}$. Then $a$ is $(b, c)$-invertible if and only if $a$ is invertible along $d$. In this case, $(b, c)$-inverse of a coincides with the inverse along $d$ of a.

The following theorem is a generalization of some well-known results of the $\{1,4\}$ inverse and the group inverse.

Theorem 3.14. Let $a, b, c \in R$. Then the following are equivalent:

(1) there exists $y \in R$ such that aya $=a$, yay $=y$ and $y R=b R$;

(2) $a$ is regular, $a R=a b R$ and $(a b)^{\circ}=b^{\circ}$;

(3) $a$ is regular and $R=a^{\circ} \oplus b R$.

Proof. (1) $\Rightarrow(3)$. If there exists $y \in R$ such that aya $=a$, yay $=y$ and $y R=b R$, then $a^{\circ} \cap b R=\{0\}$ by Lemma 2.5 and $y R=b R$. Since $1=y a+(1-y a) \in y R+a^{\circ}=b R+a^{\circ}$ by $y R=b R$ and $a y a=a$, thus $R=a^{\circ} \oplus b R$.

$(3) \Rightarrow(2)$. Suppose that $a$ is regular and $R=a^{\circ} \oplus b R$. Then $1=b r+s$ for some $r \in R$ and $s \in a^{\circ}$. Thus $a=a(b r+s)=a b r \in a b R$ by $a s=0$, which gives that $a R=a b R$. Let $t \in(a b)^{\circ}$. Then $a b t=0$ implies that $b t \in a^{\circ}$. Since $b t \in b R$, thus $b t \in b R \cap a^{\circ}=\{0\}$ by $R=a^{\circ} \oplus b R$, that is $b t=0$, thus $t \in b^{\circ}$. Therefore $(a b)^{\circ}=b^{\circ}$. 
$(2) \Rightarrow(1)$. Suppose that $a$ is regular, $a R=a b R$ and $(a b)^{\circ}=b^{\circ}$. Then by Lemma 2.4 and $a R=a b R$, we have that $a b$ is regular. By Lemma 2.3 and $a b$ is regular, we have that $(a b)^{\circ}=b^{\circ}$ implies $R b \subseteq R a b$. Let $(a b)^{-} \in(a b)\{1\}$ and $y=b(a b)^{-}$. We will check that $a y a=a, y a y=y$ and $y R=b R$. The conditions $a R=a b R$ and $a b$ is regular give that $a=a b(a b)^{-} a$, that is $a=a y a$. The conditions $R b=R a b$ and $a b$ is regular give that $b=b(a b)^{-} a b$, then $y R=b R$ by $y=b(a b)^{-}$. By $b=b(a b)^{-} a b$, we have yay $=b(a b)^{-} a b(a b)^{-}=b(a b)^{-}=y$.

Let $a, y \in R$. By the proof of [22, Theorem 3.1], we have

$$
\begin{aligned}
& a y a=a, \text { yay }=y, a y^{2}=y, y a^{2}=a \Leftrightarrow a y^{2}=y, y a^{2}=a . \\
& a y a=a, y a y=y, y^{2} a=y, a^{2} y=a \Leftrightarrow y^{2} a=y, a^{2} y=a .
\end{aligned}
$$

If we take $b=a^{*}$ in Theorem 3.14, then there exists $y \in R$ such that aya $=a$, yay $=y$ and $y R=a^{*} R$ if and only if $a \in R^{\{1,4\}}$ by Lemma 2.1. Note that the condition $a R=a a^{*} R$ or $R=a^{\circ} \oplus a^{*} R$ implies that $a$ is regular by Lemma 2.1 .

If we take $b=a$ in Theorem 3.14, It is easy to check that there exists $y \in R$ such that $a y a=a, y a y=y$ and $y R=a R$ is equivalent to aya $=a, y a y=y, a y^{2}=y$ and $y a^{2}=a$. Thus, there exists $y \in R$ such that $a y^{2}=y$ and $y a^{2}=a$ if and only if $a \in R^{\#}$ by Lemma 2.2 and (3.6).

In a similar manner, we have the following theorem, which a corresponding theorem of the Theorem 3.14. The following theorem is a generalization of some well-known results of the $\{1,3\}$-inverse and the group inverse.

Theorem 3.15. Let $a, b, c \in R$. Then the following are equivalent:

(1) there exists $y \in R$ such that aya $=a$, yay $=y$ and $R y=R c$;

(2) a is regular, $R c a=R a$ and ${ }^{\circ}(c a)={ }^{\circ} c$;

(3) a is regular and $R={ }^{\circ} a \oplus R c$.

By Theorem 3.14 and Theorem 3.15, we have the following theorem. In the following theorem, we answer the question when the $(b, c)$-inverse of $a$ is an inner inverse of $a$.

Theorem 3.16. Let $a, b, c \in R$. Then the following are equivalent:

(1) there exists $y \in R$ such that aya $=a$ and $y$ is the $(b, c)$-inverse of $a$;

(2) a is regular, $a R=a b R, R c a=R a,(a b)^{\circ}=b^{\circ}$ and ${ }^{\circ}(c a)={ }^{\circ} c$;

(3) a is regular, $R=a^{\circ} \oplus b R$ and $R={ }^{\circ} a \oplus R c$.

By Theorem 3.16 and the properties of the Moore-Penrose inverse, group inverse and core inverse, we have the following corollaries.

Corollary 3.17. Let $a \in R$. Then the following are equivalent:

(1) $a \in R^{\oplus}$;

(2) $a R=a^{2} R, R a^{*} a=R a$ and $\left(a^{2}\right)^{\circ}=a^{\circ}$;

(3) [22, Proposition 2.11] $R=a^{\circ} \oplus a R$ and $R={ }^{\circ} a \oplus R a^{*}$; 
(4) [22, Theorem 2.6] $a \in R^{\#} \cap R^{\{1,3\}}$.

Proof. It is obvious by Lemma 2.1 and Lemma 2.2 .

Corollary 3.18. Let $a, x \in R$. Then the following are equivalent:

(1) $\left[18\right.$, Theorem 2.8] $a^{\dagger}=x$ if and only if $a x a=a, x R=a^{*} R$ and $R x=R a^{*}$;

(2) $\left[18\right.$, Theorem 2.7] $a^{\#}=x$ if and only if $a x a=a, x R=a R$ and $R x=R a$.

\section{ACKNOWLEDGMENTS}

The first author is grateful to China Scholarship Council for giving him a scholarship for his further study in Universitat Politècnica de València, Spain.

\section{References}

[1] Baksalary, O.M., Trenkler G.: Core inverse of matrices. Linear Multilinear Algebra, 58, 681-697 (2010)

[2] Ben-Israel, A., Greville, T.N.E.: Generalized Inverses: Theory and Applications, 2nd ed., Springer-Verlag, New York, 2003

[3] Benítez, J., Boasso, E., Jin, H.W.: On one-sided ( $B, C)$-inverses of arbitrary matrices. arXiv:1701.09054v1, (2017)

[4] Boasso, E., Kantún-Montiel, G.: The $(b, c)$-inverses in rings and in the Banach context. Mediterr. J. Math. 14: 112. doi:10.1007/s00009-017-0910-1 (2017)

[5] Bhaskara Rao, K.R.S.: The Theory of Generalized Inverses over Commutative Rings. Taylor and Francis, London and New York, 2002

[6] Campbell, S.L., Meyer, C.D.: Generalized Inverses of Linear Transformations, Pitman, London, 1979.

[7] Drazin, M.P.: A class of outer generalized inverses. Linear Algebra Appl. 436, 1909$1923(2012)$

[8] Drazin, M.P.: Left and right generalized inverses. Linear Algebra Appl. 510, 64-78 (2016)

[9] Green, J.A.: On the structure of semigroups. Ann. Math. 54(1), 163-172 (1951)

[10] Hartwig, R.E.: Block generalized inverses. Arch. Ration. Mech. Anal. 61, 197-251 (1976)

[11] Han, R.Z., Chen, J.L.: Generalized inverses of matrices over rings. Chinese Quarterly J. Math. 7(4), 40-49 (1992)

[12] Ke, Y.Y., Cvetković-Ilić, D.S., Chen, J.L., Višnjić J.: New results on $(b, c)$-inverses. Linear Multilinear Algebra, doi.org/10.1080/03081087.2017.1301362.

[13] Ke, Y.Y., Višnjić, J., Chen, J.L.: One sided-inverse in rings. arXiv:1607.06230v1, (2016) 
[14] Mary, X.: On generalized inverse and Green's relations. Linear Algebra Appl. 434, 1836-1844 (2011)

[15] Mary, X., Patrício, P.: Generalized inverses modulo $\mathcal{H}$ in semigroups and rings. Linear Multilinear Algebra 61(8), 1130-1135 (2013)

[16] von Neumann, J.: On regular rings. Proc. Natl. Acad. Sci. U.S.A. 22(12), 707-713 (1936)

[17] Rakić, D.S.: A note on Rao and Mitra's constrained inverse and Drazin's (b,c) inverse. Linear Algebra Appl. 523, 102-108 (2017)

[18] Rakić, D.S., Dinčić, N.Č., Djordjević, D.S.: Group, Moore-Penrose, core and dual core inverse in rings with involution. Linear Algebra Appl. 463, 115-133 (2014)

[19] Rao, C.R., Mitra, S.K.: Generalized inverse of a matrix and its application. in: Proc. Sixth Berkeley Symp on Math. Statist. and Prob., 1, 601-620 (1972)

[20] Wei, Y.M.: A characterization and representation of the generalized inverse $A_{T, S}^{(2)}$ and its applications. Linear Algebra Appl. 280, 87-96 (1998)

[21] Wang, L., Chen, J.L., Castro-González, N.: Characterizations of the $(b, c)$-inverse in a ring. arXiv:1507.01446v1, (2015)

[22] Xu, S.Z., Chen, J.L., Zhang, X.X.: New characterizations for core inverses in rings with involution. Front. Math. China. 12(1), 231-246 (2017) 